\title{
Real-Time Interactions Using Virtual Tools
}

\author{
Cynthia Bruyns ${ }^{1,2}$, Steven Senger ${ }^{3}$, Simon Wildermuth ${ }^{1,2}$, \\ Kevin Montgomery ${ }^{2}$, and Richard Boyle ${ }^{1}$ \\ 1 Center for Bioinformatics, NASA Ames Research Center \\ \{cbruyns, rboyle\}@mail.arc.nasa.gov \\ 2 National Biocomputation Center, Stanford University \\ \{bruyns, wis, kevin\}@biocomp.stanford.edu \\ 3 University of Wisconsin - La Crosse, Department of Computer Science \\ senger@csfac.uwlax.edu
}

\begin{abstract}
We extend the current collision response methods to allow a user to interactively manipulate surface and volumetric meshes with virtual tools and expand the idea of interaction directions to allow the same tool to perform multiple tasks.
\end{abstract}

\section{Introduction}

We have developed a method to model complex tool and tissue interaction that can be used to model manipulations such as pushing, pulling, and cutting at multiple points of contact; and allows for several optimizations to be made within the collision detection and response schemes.

\section{Methods}

\subsection{Active Objects}

Modeled objects have well-defined borders as outlined by the geometric primitives used to represent the volume. These borders can be assigned actions and can switch which one is implemented depending on the object's state. When modeling a simple tool, such as a scalpel, one can choose a single edge to be considered sharp. Figure 1 demonstrates the two states of the sharp edge shown in green. In the first frame, the surface deforms until the force within the springs becomes larger than the yield force of the deformable tissue being simulated. In the second frame, the sharp edge cuts as the user moves the scalpel. A series of edges can also be specified as sharp in order to model more complex virtual tools such as scissors. Figure 2 demonstrates the use of multiple edges. The first frame demonstrates the tissue deformation as the scissors pinch the surface followed, in the second frame, by surface relaxation as the scissors are opened.

The selection of sharp edges automatically defines allowable cutting directions. Motion along the cut direction allows the cutting action to be implemented, while motion out of the allowed direction causes the object to perform 
the pushing action. Figure 3 demonstrates the application of this concept on a simple model. In the first frame, the user has moved the scalpel out of the cutting plane, which does not produce a cut, instead, in the second frame, we see the surface deform as the scalpel pushes the surface to the side.

\subsection{Collision Detection}

Each object within the virtual environment has a tree of hierarchical bounding spheres [1]. We can choose to speed up the collision detection by pruning the trees to capture only the important aspects of the object interactions. We can also improve performance by leveraging spatial coherence since we typically move through an object by passing over adjacent faces when manipulating a surface, and adjacent tetrahedral when manipulating a volume. We have also developed schemes for adding and removing bounding sphere leafs to the tree as a cut is being created so that entire destruction and recreation of the tree is not necessary.

\subsection{Collision Response}

Cutting occurs on each intersected element as described in [2]. Because we deal with element states and not an object-wide state, it is possible to cut complex surfaces with folds and multiple layers. Figure 4 demonstrates the ability to interact with these complex objects. In the first frame the scalpel intersects multiple layers forming multiple cut paths. In the second frame, the surfaces relax as the free edges are formed. Using the same face- based cases, we can compose overall tetrahedral cases similar to [3] using only the minimal number of new tetrahedra for each cut. Figure 5 demonstrates the ability to cut a volumetric object composed of tetrahedra.

\section{Conclusions}

We have demonstrated the ability to model different behaviors within the same virtual object by assigning actions to object borders and report initial results with this system.

Acknowledgements: This work was supported by grants to the NASA Ames Center for Bioinformatics and the National Biocomputation Center. Special thanks to Joel Brown, Fredric Mazzella, and Anil Menon.

\section{References}

1. Sorkin, S., Distance Computing Between Deformable Objects. Honors Thesis, Computer Science Department, Stanford University, (2000)

2. Bruyns, C., Senger. S., Interactive Cutting of 3D Surface Meshes. Computer and Graphics, 25 (2001) In Press

3. Mor, A., Kanade, T., Modifying Soft Tissue Models: Progressive Cutting With Minimal New Element Creation. Lecture Notes in Computer Science, Vol. 1935. Springer-Verlag, Berlin Heidelberg New York (2000) 598-607 


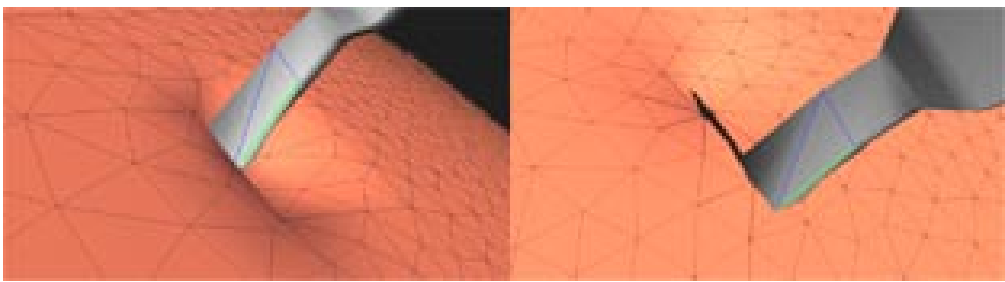

Fig. 1. Object states (a) Tool in pushing mode (b) Tool in cutting mode

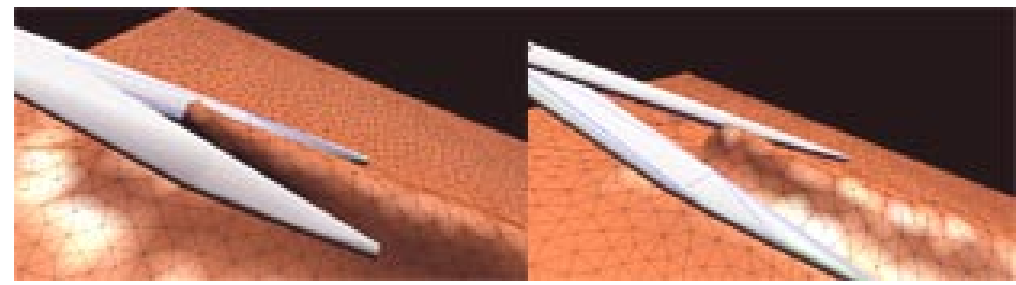

Fig. 2. Use of multiple cutting edges (a) Edges pinching surface (b) Surface relaxing

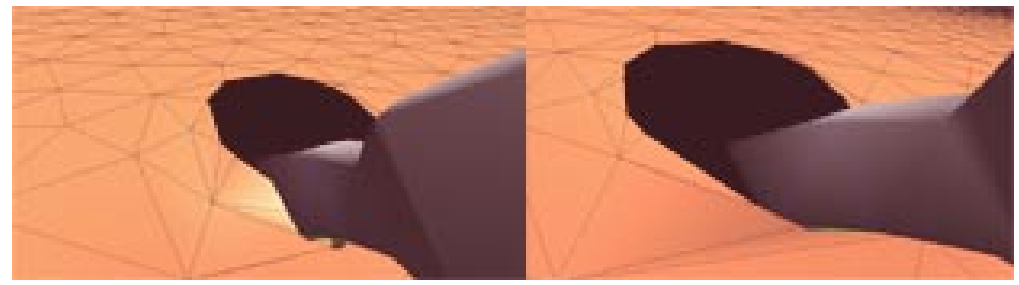

Fig. 3. Action directions a) Tool motion out of plane (b) Pushes aside surface

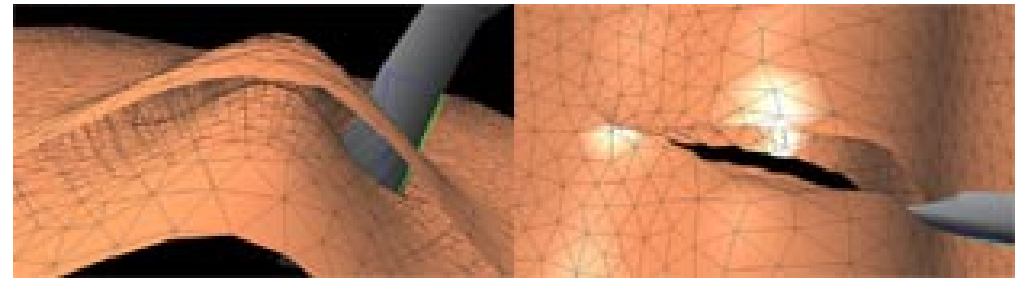

Fig. 4. Cutting multiple layers (a) Side-view (b) Top-view

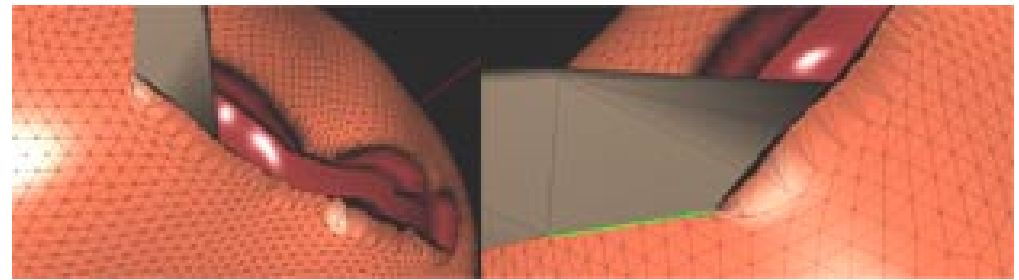

Fig. 5. Cutting a volumetric object 